Research Article

\title{
Supervariable Approach to the Nilpotent Symmetries for a Toy Model of the Hodge Theory
}

\author{
D. Shukla, ${ }^{1}$ T. Bhanja, ${ }^{1}$ and R. P. Malik ${ }^{1,2}$ \\ ${ }^{1}$ Physics Department, Centre of Advanced Studies, Banaras Hindu University, Varanasi 221 005, India \\ ${ }^{2}$ DST Centre for Interdisciplinary Mathematical Sciences, Faculty of Science, Banaras Hindu University, Varanasi 221 005, India \\ Correspondence should be addressed to T. Bhanja; tapobroto.bhanja@gmail.com
}

Received 15 March 2016; Revised 29 April 2016; Accepted 10 May 2016

Academic Editor: Elias C. Vagenas

Copyright (c) 2016 D. Shukla et al. This is an open access article distributed under the Creative Commons Attribution License, which permits unrestricted use, distribution, and reproduction in any medium, provided the original work is properly cited. The publication of this article was funded by $\mathrm{SCOAP}^{3}$.

\begin{abstract}
We exploit the standard techniques of the supervariable approach to derive the nilpotent Becchi-Rouet-Stora-Tyutin (BRST) and anti-BRST symmetry transformations for a toy model of the Hodge theory (i.e., a rigid rotor) and provide the geometrical meaning and interpretation to them. Furthermore, we also derive the nilpotent (anti-)co-BRST symmetry transformations for this theory within the framework of the above supervariable approach. We capture the (anti-)BRST and (anti-)co-BRST invariance of the Lagrangian of our present theory within the framework of augmented supervariable formalism. We also express the (anti-)BRST and (anti-)co-BRST charges in terms of the supervariables (obtained after the application of the (dual-)horizontality conditions and (anti-)BRST and (anti-)co-BRST invariant restrictions) to provide the geometrical interpretations for their nilpotency and anticommutativity properties. The application of the dual-horizontality condition and ensuing proper (i.e., nilpotent and absolutely anticommuting) fermionic (anti-)co-BRST symmetries are completely novel results in our present investigation.
\end{abstract}

\section{Introduction}

The model of a rigid rotor has played a very decisive role in unraveling some of the deepest mysteries of nature (especially in the context of atomic, molecular, and nuclear physics). This model has also been shown to be a prototype example of a gauge theory because it is endowed with the first-class constraints in the language of Dirac's prescription for classification scheme $[1,2]$. As a consequence, it has also been discussed within the framework of Becchi-RouetStora-Tyutin (BRST) formalism for its quantization and constraint analysis (see, e.g., [3] for details). We have shown, in our recent publication [4], that this toy model has a rich mathematical structure behind it because it provides a tractable physical example for the Hodge theory (within the framework of the BRST formalism) where the continuous and discrete symmetries of the theory provide the physical realizations of the de Rham cohomological operators of differential geometry (see, e.g., [5-8]).

Two key mathematical properties, associated with the (anti-)BRST symmetries (and their corresponding charges), are the nilpotency property and the absolute anticommutativity. The superfield approach to BRST formalism (see, e.g., [9-13]) provides the geometrical origin and interpretation for these abstract mathematical properties in the language of the translational generators along the Grassmannian directions of the supermanifold on which the ordinary gauge theories are generalized. This approach has been applied in the context of a rigid rotor, too, so that the geometrical basis for its (anti-)BRST symmetries could be provided (see, e.g., [4]). However, some unusual approximations have been made to derive the correct results. One of the purposes of our present investigation is to derive the nilpotent (anti-)BRST symmetry transformations in a clear fashion on the basis of physically intuitive restrictions and provide the geometrical origin for them.

As has been pointed out earlier, the model of a rigid rotor is a physical example of Hodge theory within the framework of BRST formalism. Hence, there are nilpotent (anti-)BRST and (anti-)co-BRST symmetries in the theory (besides a unique bosonic and a ghost-scale symmetry). In this context, it is a challenging problem to provide a geometrical basis 
for the (anti-)co-BRST symmetry transformations within the framework of superfield approach to BRST formalism [5-8]. We resolve this issue in our present investigation by applying the augmented version of dual-horizontality condition (DHC) and not only derive the proper nilpotent (anti-)coBRST symmetry transformations but also provide the geometrical basis for their existence in the same manner as that of the (anti-)BRST symmetries (which has already been done in our earlier work [4]). In the application of the DHC, we exploit the working-rule, established in [14], for the Hodge duality $\star$ operation on a given supermanifold and obtain the precise results which establishes the correctness of the rules which have been laid down in our earlier publication [14].

In our present investigation, we have also provided the geometrical basis for the nilpotency and absolute anticommutativity of the (anti-)co-BRST charges (on the same lines as we have provided for the (anti-)BRST charges in our earlier work [4]). Furthermore, we also capture the (anti-)BRST and (anti-)co-BRST invariance of the Lagrangian of our present theory within the framework of the augmented version of supervariable approach. This exercise leads to the geometrical interpretation for the (anti-)BRST and (anti-)co-BRST invariance of the Lagrangian in the language of the translation of a specific sum of composite supervariables (obtained after the appropriate set of restrictions) along the Grassmannian directions of the chosen supermanifold on which our ordinary theory is generalized within the framework of the supervariable approach to BRST formalism.

Our present endeavor is essential on the following counts. First, in our earlier work [4], we have made some approximations to obtain the proper (anti-)BRST symmetry transformations within the framework of augmented supervariable approach. Thus, it is essential for us to derive the same symmetry transformations in a physically intuitive manner by exploiting the horizontality condition and (anti-)BRST invariant restrictions. We have accomplished this goal in our present endeavor. Second, to put the idea of the dualhorizontality condition (DHC) on the firmer footings, it is necessary for us to apply it to our present system and derive the proper (anti-)co-BRST symmetry transformations. We have obtained these symmetry transformations in a consistent manner by exploiting the idea of DHC. Finally, it is challenging for us to provide the geometrical basis for the nilpotent (anti-)co-BRST transformations (and corresponding generators) within the framework of the supervariable approach (as has already been done in [4] for the (anti-)BRST symmetries and their generators). We have achieved this goal, too, in our present endeavor.

The material of our present paper is organized as follows. In Section 2, we briefly mention about the nilpotent (anti-) BRST and (anti-)co-BRST symmetries for the Lagrangian of our present theory. Section 3 is devoted to the derivation of nilpotent (anti-)BRST symmetries within the framework of augmented supervariable formalism. Section 4 of our present endeavor contains the application of dual-horizontality condition and the derivation of the (anti-)co-BRST symmetries. Section 5 is devoted to capturing the geometrical meaning of the invariance of Lagrangian of our present theory under
(anti-)BRST and (anti-)co-BRST transformations. In Section 6 , we discuss the geometrical meaning of the nilpotency property of the (anti-)BRST and (anti-)co-BRST charges by expressing them in terms of the supervariables (obtained after various appropriate restrictions). Finally, we make some concluding remarks and point out a few future directions in Section 7.

In our Appendix, we perform an explicit computation which is used in the main body of our text in the context of application of the dual-horizontality condition (DHC).

\section{Preliminaries: Lagrangian and Symmetries}

We begin with the following (anti-)BRST and (anti-)co-BRST invariant first-order Lagrangian for the rigid rotor (see, e.g., $[3,4]$ for details):

$$
\begin{aligned}
L_{b}= & \dot{r} p_{r}+\dot{\vartheta} p_{\vartheta}-\frac{p_{\vartheta}^{2}}{2 r^{2}}-\lambda(r-a)+b\left(\dot{\lambda}-p_{r}\right)+\frac{b^{2}}{2} \\
& -i \dot{\bar{C}} \dot{C}+i \bar{C} C
\end{aligned}
$$

where $r$ and $\vartheta$ are the polar coordinates and their corresponding generalized velocities are $\dot{r}$ and $\dot{\vartheta}$. The momenta for the particle (of mass $m=1$ ), moving on a circle of radius $a$, are $p_{r}$ and $p_{9}$. Here $\lambda$ is a Lagrange multiplier that turns out to be the "gauge" variable of our present theory. The variable $b$ is the Nakanishi-Lautrup type of auxiliary variable and $(\bar{C}) C$ are the (anti)ghost fermionic $\left(C^{2}=\bar{C}^{2}=0, C \bar{C}+\bar{C} C=0\right)$ variables. All these variables are function of the evolution parameter $t$ and an overdot on the variables always denotes the derivative with respect to it (i.e., $\dot{\vartheta}=d \vartheta / d t, \dot{\lambda}=d \lambda / d t$ ).

We observe that, under the following nilpotent $\left(s_{b}^{2}=\right.$ $\left.s_{a b}^{2}=0\right)$ and absolutely anticommuting $\left(s_{b} s_{a b}+s_{a b} s_{b}=0\right)$ continuous (anti-)BRST symmetry transformations $\left(s_{(a) b}\right)$

$$
\begin{aligned}
s_{b} \lambda & =\dot{C}, \\
s_{b} \bar{C} & =i b, \\
s_{b} p_{r} & =-C, \\
s_{b}\left[C, r, \vartheta, p_{\vartheta}, b\right] & =0, \\
s_{a b} \lambda & =\dot{\bar{C}}, \\
s_{a b} C & =-i b, \\
s_{a b} p_{r} & =-\bar{C}, \\
s_{a b}\left[\bar{C}, r, \vartheta, p_{\vartheta}, b\right] & =0,
\end{aligned}
$$

the Lagrangian $L_{b}$ transforms to the total time derivatives:

$$
\begin{aligned}
s_{a b} L_{b} & =\frac{d}{d t}[b \dot{\bar{C}}-\bar{C}(r-a)], \\
s_{b} L_{b} & =\frac{d}{d t}[b \dot{C}-C(r-a)],
\end{aligned}
$$


thereby rendering the action integral $S=\int d t L_{b}$ invariant. Hence, transformations (2) are the symmetry transformations for the action $S$. There are other nilpotent $\left(s_{d}^{2}=s_{a d}^{2}=0\right)$ and absolutely anticommuting $\left(s_{d} s_{a d}+s_{a d} s_{d}=0\right)$ symmetries in the theory. These (anti-)co-BRST [or (anti-)dual-BRST] symmetry transformations $\left(s_{(a) d}\right)$ are

$$
\begin{aligned}
s_{d} \lambda & =\bar{C}, \\
s_{d} C & =i(r-a), \\
s_{d} p_{r} & =\dot{\bar{C}}, \\
s_{d}\left[\bar{C}, r, \vartheta, p_{\vartheta}, b\right] & =0, \\
s_{a d} \lambda & =C, \\
s_{a d} \bar{C} & =-i(r-a), \\
s_{a d} p_{r} & =\dot{C}, \\
s_{a d}\left[C, r, \vartheta, p_{\vartheta}, b\right] & =0,
\end{aligned}
$$

leaving the Lagrangian absolutely invariant (i.e., $s_{(a) d} L_{b}=0$ ).

We have demonstrated that the action integral $S=\int d t L_{b}$ and Lagrangian $\left(L_{b}\right)$ remain invariant under the continuous (anti-)BRST and (anti-)co-BRST symmetry transformations, respectively. Thus, according to Noether's theorem, the following conserved and nilpotent (anti-)BRST $\left(Q_{(a) b}\right)$ and (anti-)co-BRST $\left(Q_{(a) d}\right)$ charges, namely,

$$
\begin{aligned}
Q_{b} & =b \dot{C}-\dot{b} C, \\
Q_{a b} & =b \dot{\bar{C}}-\dot{b} \bar{C}, \\
Q_{d} & =b \bar{C}+\dot{b} \dot{\bar{C}}, \\
Q_{a d} & =b C+\dot{b} \dot{C},
\end{aligned}
$$

are the generators for the (anti-)BRST and (anti-)co-BRST symmetry transformations, as it can be explicitly checked that

$$
s_{r} \phi= \pm i\left[\phi, Q_{r}\right]_{ \pm}, \quad r=b, a b, d, a d,
$$

for the generic variable $\phi=r, \vartheta, p_{r}, p_{\vartheta}, \lambda, b, C, \bar{C}$. Here \pm signs, as the subscripts on the square bracket, correspond to the (anti)commutator for the generic variable $\phi$ of our theory being (fermionic) bosonic in nature.

We wrap up this section with the following remarks. First, under the (anti-)BRST symmetry transformations, it is the kinetic term $\left[\left(\dot{\vartheta}_{p_{\vartheta}}\right)-\left(p_{\vartheta}^{2} / 2 r^{2}\right)\right]=(1 / 2) \dot{r}^{2} \dot{\vartheta}^{2}=(1 / 2) v^{2}$ that remains invariant. Second, the gauge-fixing term $(\dot{\lambda}-$ $p_{r}$ ) turns out to be invariant quantity under the nilpotent (anti-)co-BRST symmetry transformations. Third, the kinetic term $(1 / 2) v^{2}$ has its origin [4] in the exterior derivative $d=$ $d t \partial_{t}$ (with $\left.d^{2}=0\right)$. Fourth, the gauge-fixing term $\left(\dot{\lambda}-p_{r}\right)$ owes its origin to the coexterior derivative $\delta=* d *\left(\right.$ with $\delta^{2}=0$ ) of differential geometry [4] where $*$ is the Hodge duality operation. Fifth, the anticommutator of the (anti-)BRST and (anti-)co-BRST symmetry transformations defines a unique bosonic symmetry in the theory which corresponds to the Laplacian operator of differential geometry. Finally, the present toy model of a rigid rotor which turns out to be the physical example of a Hodge theory within the framework of BRST formalism [4].

\section{3. (Anti-)BRST Symmetries: Supervariable Formalism}

In our earlier work [4], the (anti-)BRST symmetry transformations have been obtained by exploiting the basic ideas of supervariable formalism. However, there have been adhoc assumptions and approximations in deriving the correct results. In our present section, we exploit the horizontality condition and (anti-)BRST invariant restriction to obtain the appropriate (anti-)BRST symmetry transformations for our system without making any approximations. Our method of derivation is simpler and physically more intuitive. To corroborate these statements, first of all, we generalize the gauge and (anti)ghost variables (i.e., $\lambda(t), C(t), \bar{C}(t))$ onto $(1,2)$-dimensional supermanifold as supervariables:

$$
\begin{gathered}
\lambda(t) \longrightarrow \Lambda(t, \theta, \bar{\theta})=\lambda(t)+\theta \bar{R}(t)+\bar{\theta} R(t)+i \theta \bar{\theta} S(t), \\
C(t) \longrightarrow F(t, \theta, \bar{\theta})=C(t)+i \theta \bar{B}_{1}(t)+i \bar{\theta} B_{1}(t)+i \theta \bar{\theta} s(t), \\
\bar{C}(t) \longrightarrow \bar{F}(t, \theta, \bar{\theta})=\bar{C}(t)+i \theta \bar{B}_{2}(t)+i \bar{\theta} B_{2}(t)+i \theta \bar{\theta} \bar{s}(t),
\end{gathered}
$$

where the expansions have been made along the Grassmannian directions $(\theta, \bar{\theta})$ of the $(1,2)$-dimensional supermanifold which is parametrized by the superspace variable $Z^{M}=$ $(t, \theta, \bar{\theta})$ and the secondary variables $(R, \bar{R}, s, \bar{s})$ are fermionic and $\left(B_{1}, \bar{B}_{1}, B_{2}, \bar{B}_{2}, S\right)$ are bosonic in nature. It is elementary to check that, in the limit $\theta=0, \bar{\theta}=0$, we get back the original variables $(\lambda(t), C(t), \bar{C}(t))$ of our starting Lagrangian (1). We christen the above supersymmetric generalized variables as "supervariables" (and not superfields) because, in the limit $\theta=$ $\bar{\theta}=0$, we retrieve back our basic dynamical variables (and not the fields).

In one $(0+1)$-dimensional ordinary space, we note that the 1-forms $d=d t \partial_{t}, \lambda^{(1)}=d t \lambda(t)$, lead to the definition of a 2-form $d \lambda^{(1)}=(d t \wedge d t) \dot{\lambda}=0$, where $d=d t \partial_{t}$ is the exterior derivative (with $\left.d^{2}=0\right)$ and $(d t \wedge d t)=0$. These operators can be generalized onto $(1,2)$-dimensional supermanifold to their supersymmetric counterparts as

$$
\begin{aligned}
& d \longrightarrow \widetilde{d}=d Z^{M} \partial_{M} \equiv d t \partial_{t}+d \theta \partial_{\theta}+d \bar{\theta} \partial_{\bar{\theta}}, \quad \widetilde{d}^{2}=0, \\
& \lambda^{(1)} \longrightarrow \tilde{\lambda}^{(1)}=d Z^{M} A_{M} \equiv d t \Lambda(t, \theta, \bar{\theta})+d \theta \bar{F}(t, \theta, \bar{\theta})+d \bar{\theta} F(t, \theta, \bar{\theta}),
\end{aligned}
$$


where the supervariables $(\Lambda(t, \theta, \bar{\theta}), F(t, \theta, \bar{\theta}), \bar{F}(t, \theta, \bar{\theta}))$ form a vector supermultiplet $A_{M}$ on the (1,2)-dimensional supermanifold whose expansions along the Grassmannian directions have been given in (7). In the above, we have taken $\partial_{M}=\partial / \partial Z^{M} \equiv\left(\partial_{t}, \partial_{\theta}, \partial_{\bar{\theta}}\right)$ as the derivatives with respect to the evolution parameter $t$ and the Grassmannian variables $(\theta, \bar{\theta})$. The super 2-form, constructed with $\tilde{d}$ and $\tilde{\lambda}^{(1)}$, has the following explicit form:

$$
\begin{aligned}
\tilde{d} \tilde{\lambda}^{(1)}= & (d t \wedge d t)\left(\partial_{t} \bar{F}-\partial_{\theta} \Lambda\right)+(d t \wedge d \bar{\theta})\left(\partial_{t} F-\partial_{\bar{\theta}} \Lambda\right) \\
& +(d \theta \wedge d \theta)\left(\partial_{\theta} \bar{F}\right)+(d \bar{\theta} \wedge d \bar{\theta})\left(\partial_{\bar{\theta}} F\right) \\
& +(d \theta \wedge d \bar{\theta})\left(\partial_{\theta} F+\partial_{\bar{\theta}} \bar{F}\right) .
\end{aligned}
$$

The horizontality condition requires that $d \lambda^{(1)}=\tilde{d} \widetilde{\lambda}^{(1)}=0$. Thus, we obtain the following expressions for the secondary variables in terms if the basic dynamical and auxiliary variables; namely (see, e.g., [4] for details),

$$
\begin{aligned}
R & =\dot{C}, \\
\bar{R} & =\dot{\bar{C}}, \\
S & =\dot{b}, \\
\bar{B}_{2} & =0, \\
B_{1} & =0, \\
s & =0, \\
\bar{B}_{1}+B_{2} & =0, \\
\bar{s} & =0 .
\end{aligned}
$$

The condition $\bar{B}_{1}+B_{2}=0$ is nothing but the Curci-Ferrari type restriction which is trivial in our case. Thus, we choose $B_{2}=-\bar{B}_{1}=b$. This specific choice can be derived using the (anti-)BRST invariant restriction, too. As a consequence, we have the following expansions for the supervariables after the application of the horizontality condition ( $\mathrm{HC})$ :

$$
\begin{aligned}
\Lambda^{(h)}(t, \theta, \bar{\theta})= & \lambda(t)+\theta(\dot{\bar{C}})+\bar{\theta}(\dot{C})+\theta \bar{\theta}(i \dot{b}) \\
\equiv & \lambda(t)+\theta\left(s_{a b} \lambda\right)+\bar{\theta}\left(s_{b} \lambda\right) \\
& +\theta \bar{\theta}\left(s_{b} s_{a b} \lambda\right) \\
F^{(h)}(t, \theta, \bar{\theta})= & C(t)+\theta(-i b)+\bar{\theta}(0)+\theta \bar{\theta}(0) \\
\equiv & C(t)+\theta\left(s_{a b C}\right)+\bar{\theta}\left(s_{b} C\right) \\
& +\theta \bar{\theta}\left(s_{b} s_{a b} C\right) \\
\bar{F}^{(h)}(t, \theta, \bar{\theta})= & \bar{C}(t)+\theta(0)+\bar{\theta}(i b)+\theta \bar{\theta}(0) \\
\equiv & \bar{C}(t)+\theta\left(s_{a b} \bar{C}\right)+\bar{\theta}\left(s_{b} \bar{C}\right) \\
& +\theta \bar{\theta}\left(s_{b} s_{a b} \bar{C}\right),
\end{aligned}
$$

where the superscript $(h)$, on the supervariables, denotes the superexpansions, obtained after the application of the HC. It is evident, from the above, that we have already derived the (anti-)BRST symmetry transformations for the variables $(\lambda(t), C(t), \bar{C}(t))$.

To derive the (anti-)BRST symmetries for the momentum variable $p_{r}(t)$, we have to exploit the (anti-)BRST invariant restrictions (BIRs). In this context, we note that the (anti-)BRST invariant quantity (i.e., quantity present in the square bracket),

$$
s_{(a) b}\left[b(t) p_{r}(t)-i \bar{C}(t) C(t)\right]=0,
$$

can be generalized onto the $(1,2)$-dimensional supermanifold as

$$
B(t, \theta, \bar{\theta}) P_{r}(t, \theta, \bar{\theta})-i \bar{F}^{(h)}(t, \theta, \bar{\theta}) F^{(h)}(t, \theta, \bar{\theta}) .
$$

The (anti-)BRST invariance of the Nakanishi-Lautrup auxiliary variable $b(t)$ [i.e., $s_{(a) b} b(t)=0$ ] implies that $b(t) \rightarrow$ $B(t, \theta, \bar{\theta})=b(t)$. In other words, the supervariable $B(t, \theta, \bar{\theta})$ would have no expansion along the Grassmannian directions $(\theta, \bar{\theta})$. To proceed further, we take the general expansion for the supervariable $P_{r}(t, \theta, \bar{\theta})$ as

$$
P_{r}(t, \theta, \bar{\theta})=p_{r}(t)+\theta \bar{K}(t)+\bar{\theta} K(t)+i \theta \bar{\theta} L(t),
$$

and we demand that the (anti-)BRST invariant quantity ( $b p_{r}-$ $i \bar{C} C)$ should remain independent of the "soul" coordinates $(\theta, \bar{\theta})$. It will be noted that, in the old literature on superfield approach to BRST formalism [13], the bosonic coordinates have been christened as the "body" coordinates and Grassmannian coordinates have been called the "soul" coordinates because the latter are very abstract and cannot be physically realized in the ordinary space. In other words, we impose the following restriction:

$$
\begin{aligned}
b(t) & P_{r}(t, \theta, \bar{\theta})-i \bar{F}^{(h)}(t, \theta, \bar{\theta}) F^{(h)}(t, \theta, \bar{\theta}) \\
= & b(t) p_{r}(t)-i \bar{C}(t) C(t),
\end{aligned}
$$

which leads to the determination of the secondary variables of (14) in terms of the basic and auxiliary variables of our present theory as

$$
\begin{aligned}
\bar{K} & =-\bar{C}, \\
K & =-C, \\
L & =-b .
\end{aligned}
$$

The above results establish the bosonic nature of $L$ and fermionic nature of $(K, \bar{K})$. Thus, we have the following expansions for $P_{r}(t, \theta, \bar{\theta})$ :

$$
\begin{aligned}
P_{r}^{(b)}(t, \theta, \bar{\theta})= & p_{r}(t)+\theta(-\bar{C})+\bar{\theta}(-C)+\theta \bar{\theta}(-i b) \\
\equiv & p_{r}(t)+\theta\left(s_{a b} p_{r}\right)+\bar{\theta}\left(s_{b} p_{r}\right) \\
& +\theta \bar{\theta}\left(s_{b} s_{a b} p_{r}\right),
\end{aligned}
$$


where the superscript $(b)$ stands for the supervariable obtained after the application of the (anti-)BRST invariant restriction (15). It is evident that we have found out the (anti-)BRST symmetry transformations for $p_{r}(t)$, as

$$
\begin{gathered}
s_{b} p_{r}=-C, \\
s_{a b} p_{r}=-\bar{C}, \\
s_{b} s_{a b} p_{r}=-i b .
\end{gathered}
$$

We conclude that, for the derivation of the correct and complete set of (anti-)BRST symmetries, we have to exploit the HC and BIR (cf. (15)) together.

We close this section with the remarks that we have obtained the proper (anti-)BRST symmetry transformations for all the variables $\left(\lambda, C, \bar{C}, p_{r}\right)$ of our theory. Our method of derivation of these (anti-)BRST symmetries is more physical in content than the same derivation carried out in our earlier work [4]. The key ideas that have been exploited together in our present endeavor are the HC and (anti-)BRST invariant restrictions (BIRs) which lead to the derivation of the full set of proper (anti-)BRST symmetries. A close look at expansions (11) and (17) demonstrates that

$$
\begin{aligned}
& \left.\frac{\partial}{\partial \theta} \Omega^{(h, b)}(t, \theta, \bar{\theta})\right|_{\bar{\theta}=0}=s_{a b} \omega(t), \\
& \left.\frac{\partial}{\partial \bar{\theta}} \Omega^{(h, b)}(t, \theta, \bar{\theta})\right|_{\theta=0}=s_{b} \omega(t),
\end{aligned}
$$

where $\omega(t)$ is the ordinary one $(0+1)$-dimensional variable and $\Omega^{(h, b)}(t, \theta, \bar{\theta})$ are the supervariables obtained after $\mathrm{HC}$ and BIRs (cf. (11), (17)). The above relationships provide the geometrical meaning for the (anti-)BRST symmetry transformations $s_{(a) b}$. It states that the (anti-)BRST symmetry transformations $s_{(a) b}$. of an ordinary variable $\omega(t)$ is equivalent to the translations of the corresponding supervariable (cf. (11), (17)) along the Grassmannian directions $(\theta, \bar{\theta})$ of the $(1,2)$-dimensional supermanifold. The nilpotency of $s_{(a) b}$ (i.e., $\left.s_{(a) b}^{2}=0\right)$ is connected with two successive translations (i.e., $\left.\partial_{\theta}^{2}=0, \partial_{\bar{\theta}}^{2}=0\right)$ along the Grassmannian directions $(\theta, \bar{\theta})$ of our chosen $(1,2)$-dimensional supermanifold.

\section{Nilpotent (Anti-)Co-BRST Symmetries: Supervariable Approach}

In our present section, we will exploit the concept of dualhorizontality condition to derive the nilpotent (anti-)coBRST symmetry transformations (cf. Section 2). The latter are characterized by the key observation that the gauge-fixing term $\left(\dot{\lambda}-p_{r}\right)$ remains invariant under it. Thus, it is clear from the key ideas of the augmented version of the supervariable approach that this quantity would remain independent of the "soul" coordinates $(\theta, \bar{\theta})$ when it is generalized onto the $(1,2)$ dimensional supermanifold. Towards this goal in mind, first of all, we note that the following is true:

$$
\delta \lambda^{(1)}=* d * \lambda^{(1)}=\dot{\lambda},
$$

where $* d *$ is the coexterior derivative, $\lambda^{(1)}=d t \lambda(t)$ is the 1 form in one $(0+1)$-dimensional ordinary space, and $(*)$ is the Hodge duality operation on $1 \mathrm{D}$ ordinary spacetime manifold. The invariance of the gauge-fixing term under the (anti-)coBRST symmetry transformations can be translated into the following (anti-)co-BRST invariant restriction (CBIR) on the supervariables of the $(1,2)$-dimensional supermanifold:

$$
\star \widetilde{d} \star \tilde{\lambda}^{(1)}-P_{r}(t, \theta, \bar{\theta})=* d * \lambda^{(1)}-p_{r}(t),
$$

where $(\star)$ is the Hodge duality operation on the $(1,2)$ dimensional supermanifold and $\tilde{\lambda}^{(1)}, \tilde{d}, P_{r}(t, \theta, \bar{\theta})$ are defined in (8) and (14). We christen the CBIR (21) as the dualhorizontality condition (DHC) because it is the coexterior derivative of differential geometry that plays a key role in the above restriction.

We have the step-by-step computation of $\star \widetilde{d} \star \tilde{\lambda}^{(1)}$ in our Appendix. Ultimately, the DHC (cf. (21)) leads to the following equality:

$$
\begin{aligned}
(\dot{\Lambda}+ & \left.\partial_{\theta} \bar{F}+\partial_{\bar{\theta}} F\right)+s^{\theta \theta}\left(\partial_{\theta} F\right)+s^{\bar{\theta} \bar{\theta}}\left(\partial_{\bar{\theta}} \bar{F}\right) \\
& -\left[p_{r}(t)+\theta \bar{K}(t)+\bar{\theta} K(t)+i \theta \bar{\theta} L(t)\right] \\
= & \dot{\lambda}-p_{r}(t) .
\end{aligned}
$$

It is clear that the coefficients of $s^{\theta \theta}$ and $s^{\bar{\theta}}$ would be zero because there are no such terms on the r.h.s. Thus, we have the following results:

$$
\begin{aligned}
\partial_{\theta} F & =0, \\
\partial_{\bar{\theta}} \bar{F} & =0, \\
\Downarrow & \\
\bar{B}_{1} & =0, \\
B_{2} & =0, \\
s & =0, \\
\bar{s} & =0 .
\end{aligned}
$$

The above values imply that the reduced form of the expansions for $F(t, \theta, \bar{\theta})$ and $\bar{F}(t, \theta, \bar{\theta})($ cf. (7)) are as given below:

$$
\begin{aligned}
& F^{(r)}(t, \theta, \bar{\theta})=C(t)+i \bar{\theta} B_{1}(t), \\
& \bar{F}^{(r)}(t, \theta, \bar{\theta})=\bar{C}(t)+i \theta \bar{B}_{2}(t) .
\end{aligned}
$$

Plugging in these expansions and that of $\Lambda(t, \theta, \bar{\theta})$ from (7) into the CBIR (cf. (21)), we obtain the following conditions on the secondary variables:

$$
\begin{aligned}
B_{1}+\bar{B}_{2} & =0, \\
\bar{K} & =\dot{\bar{R}}, \\
K & =\dot{R}, \\
L & =\dot{S} .
\end{aligned}
$$


In the above, the condition $B_{1}+\bar{B}_{2}=0$ is the analogue of Curci-Ferrari restriction. Making the choice $B_{1}(t)=\mathscr{B}$, we get $\bar{B}_{2}(t)=-\mathscr{B}$. Substitution of these values into expansions (7) and (24) leads to the following rereduced form of these expansions:

$$
\begin{aligned}
& F^{(R)}(t, \theta, \bar{\theta})=C(t)+i \bar{\theta} \mathscr{B}, \\
& \bar{F}^{(R)}(t, \theta, \bar{\theta})=\bar{C}(t)-i \theta \mathscr{B}, \\
& P^{(R)}(t, \theta, \bar{\theta})=p_{r}(t)+\theta(\dot{\bar{R}})+\bar{\theta}(\dot{R})+i \theta \bar{\theta}(\dot{S}) .
\end{aligned}
$$

The above expansions show that we have not yet found the explicit expressions for the secondary variables in terms of the basic and auxiliary variables.

The additional restrictions come from the following observations:

$$
\begin{aligned}
s_{(a) d}\left[\dot{r} p_{r}-i \dot{\bar{C}} \dot{C}\right] & =0, \\
s_{(a) d}[\lambda(r-a)-i \bar{C} C] & =0 .
\end{aligned}
$$

The above (anti-)dual-BRST invariant quantities (which are present in the square brackets) can be generalized onto $(1,2)$-dimensional supermanifold. By exploiting the idea of augmented version of supervariable approach, we have to demand that such invariant quantities should be independent of the Grassmannian variables $\theta$ and $\bar{\theta}$. Thus, we have the following equality conditions on the supervariables of our chosen supermanifold:

$$
\begin{aligned}
& \dot{R}(t, \theta, \bar{\theta}) P_{r}^{(R)}(t, \theta, \bar{\theta}) \\
& -i \dot{\bar{F}}^{(R)}(t, \theta, \bar{\theta}) \dot{F}^{(R)}(t, \theta, \bar{\theta})=\dot{r} p_{r}-i \dot{\bar{C}} \dot{C}, \\
& \Lambda(t, \theta, \bar{\theta})[R(t, \theta, \bar{\theta})-a] \\
& \quad-i \bar{F}^{(R)}(t, \theta, \bar{\theta}) F^{(R)}(t, \theta, \bar{\theta})=\lambda(r-a)-i \bar{C} C,
\end{aligned}
$$

where the expressions for $\left(P_{r}^{(r)}, F^{(R)}, \bar{F}^{(R)}\right)$ are given in (26) and $R(t, \theta, \bar{\theta})$ is the generalization of $r(t)$ onto $(1,2)$-dimensional supermanifold. However, as we know that $r(t)$ is an (anti-)co-BRST invariant (i.e., $s_{(a) d} r(t)=0$ ) quantity, we find that $R(t, \theta, \bar{\theta})=r(t)$. Plugging in these values into (28) and $\Lambda(t, \theta, \bar{\theta})$ from (7), the above equality becomes

$$
\begin{aligned}
& \dot{r}\left[p_{r}+\theta(\dot{\bar{R}})+\bar{\theta}(\dot{R})+i \theta \bar{\theta}(\dot{S})\right] \\
& \quad-i[\dot{\bar{C}}-i \theta \dot{\mathscr{B}}][\dot{C}+i \bar{\theta} \dot{\mathscr{B}}]=\dot{r} p_{r}-i \dot{\bar{C}} \dot{C}, \\
& {[\lambda+\theta \bar{R}+\bar{\theta} R+i \theta \bar{\theta} S](r-a)} \\
& \quad-i[\bar{C}-i \theta \mathscr{B}][C+i \bar{\theta} \mathscr{B}]=\lambda(r-a)-i \bar{C} C .
\end{aligned}
$$

The above two equations in (29) yield the following beautiful relationships:

$$
\begin{aligned}
\dot{\mathscr{B}} \dot{C} & =\dot{\bar{R}} \dot{r}, \\
\dot{\mathscr{B}} \dot{\bar{C}} & =\dot{R} \dot{r}, \\
\mathscr{\mathscr { B }} \mathscr{B} & =\dot{S} \dot{r}, \\
\mathscr{B} C & =\bar{R}(r-a), \\
\mathscr{B} \bar{C} & =R(r-a), \\
\mathscr{B} \mathscr{B} & =S(r-a) .
\end{aligned}
$$

Even after the relations in (30), we have not found the precise expressions for the secondary variables in terms of the basic and auxiliary variables of the theory. Thus, we have to look for other (anti-)co-BRST invariant quantities of our present theory.

We note that $s_{d}[\lambda \bar{C}]=0$ and $s_{a d}[\lambda C]=0$. These coBRST and anti-co-BRST invariant quantities would also be independent of the "soul" coordinates $\theta$ and $\bar{\theta}$ when they are generalized onto the $(1,2)$-dimensional supermanifold. Thus, we have the following restrictions on the supervariables of the above supermanifold:

$$
\begin{aligned}
& \Lambda \bar{F}^{(R)}=\lambda \bar{C}, \\
& \Lambda F^{(R)}=\lambda C .
\end{aligned}
$$

Plugging in expansions from (7) and (26), we obtain the following relationships:

$$
\begin{aligned}
R \bar{C} & =0, \\
R \mathscr{B} & =S \bar{C}, \\
\bar{R} & =i \lambda \mathscr{B}, \\
\bar{R} C & =0, \\
\bar{R} \mathscr{B} & =S C, \\
R C & =-i \lambda \mathscr{B} .
\end{aligned}
$$

The above relationships fix the value of $R$ and $\bar{R}$ as $R \propto \bar{C}$ and $\bar{R} \propto C$. We make one of the simplest choices for the secondary variables as $R=\bar{C}$ and $\bar{R}=C$. Once we make this simple choice, the rest of the secondary variables of the superexpansion also get fixed.

A careful observation of the above relationships (30) and (32) leads to the following expressions for the secondary variables in terms of basic variables of our theory:

$$
\begin{aligned}
\bar{R} & =C, \\
R & =\bar{C}, \\
\mathscr{B} & =(r-a), \\
S & =(r-a) \equiv \mathscr{B} .
\end{aligned}
$$


Substitution of these values into expansions (7) and (26) leads to the following:

$$
\begin{aligned}
\Lambda^{(d)}(t, \theta, \bar{\theta})= & \lambda(t)+\theta(C)+\bar{\theta}(\bar{C})+\theta \bar{\theta}[i(r-a)] \\
\equiv & \lambda(t)+\theta\left(s_{a d} \lambda\right)+\bar{\theta}\left(s_{d} \lambda\right) \\
& +\theta \bar{\theta}\left(s_{d} s_{a d} \lambda\right), \\
F^{(d)}(t, \theta, \bar{\theta})= & C(t)+\theta(0)+\bar{\theta}[i(r-a)]+\theta \bar{\theta}(0) \\
\equiv & C(t)+\theta\left(s_{a d} C\right)+\bar{\theta}\left(s_{d} C\right) \\
& +\theta \bar{\theta}\left(s_{d} s_{a d} C\right), \\
\bar{F}^{(d)}(t, \theta, \bar{\theta})= & \bar{C}(t)+\theta[-i(r-a)]+\bar{\theta}(0)+\theta \bar{\theta}(0) \\
\equiv & \bar{C}(t)+\theta\left(s_{a d} \bar{C}\right)+\bar{\theta}\left(s_{d} \bar{C}\right) \\
& +\theta \bar{\theta}\left(s_{d} s_{a d} \bar{C}\right), \\
P_{r}^{(d)}(t, \theta, \bar{\theta})= & p_{r}(t)+\theta(\dot{C})+\bar{\theta}(\dot{\bar{C}})+\theta \bar{\theta}(i \dot{r}) \\
\equiv & p_{r}(t)+\theta\left(s_{a d} p_{r}\right)+\bar{\theta}\left(s_{d} p_{r}\right) \\
& +\theta \bar{\theta}\left(s_{d} s_{a d} p_{r}\right),
\end{aligned}
$$

where the superscript $(d)$ denotes the expansion of the supervariables after the application of DHC. We point out that we have already derived the nilpotent and absolutely anticommuting (anti-)co-BRST symmetry transformations (4) in the above superexpansions. We note, from the above expressions, that there is a deep connection between the (anti-)coBRST symmetry transformations $s_{(a) d}$ and the translational generators $\left(\partial_{\theta}, \partial_{\bar{\theta}}\right)$ along the Grassmannian directions of the $(1,2)$-dimensional supermanifold on which our theory has been generalized. In fact, we have the following mappings:

$$
\begin{aligned}
& \left.\frac{\partial}{\partial \theta} \Sigma^{(d)}(t, \theta, \bar{\theta})\right|_{\bar{\theta}=0}=s_{a d} \sigma(t), \quad \frac{\partial}{\partial \theta} \Longleftrightarrow s_{a d}, \\
& \left.\frac{\partial}{\partial \bar{\theta}} \Sigma^{(d)}(t, \theta, \bar{\theta})\right|_{\theta=0}=s_{d} \sigma(t), \quad \frac{\partial}{\partial \bar{\theta}} \Longleftrightarrow s_{d}, \\
& \frac{\partial}{\partial \bar{\theta}} \frac{\partial}{\partial \theta} \Sigma^{(d)}(t, \theta, \bar{\theta})=s_{d} s_{a d} \sigma(t), \quad \frac{\partial}{\partial \bar{\theta}} \frac{\partial}{\partial \theta} \Longleftrightarrow s_{d} s_{a d},
\end{aligned}
$$

where $\sigma(t)$ is the generic variable of 1D ordinary space and $\Sigma^{(d)}(t, \theta, \bar{\theta})$ is the generic supervariable that is obtained in (34) with full superexpansions.

Geometrically, we note that the co-BRST symmetry transformations on a given variable $\sigma(t)$ of the $1 \mathrm{D}$ theory is equivalent to the translation of the corresponding supervariable $\Sigma^{(d)}(t, \theta, \bar{\theta})$ along the $\bar{\theta}$ direction of the supermanifold (where the Grassmannian direction $\theta$ is kept untouched). Similarly, the geometrical origin and interpretation for the anti-co-BRST symmetry transformation can be provided. We further lay emphasis on the observation that the nilpotency $\left(s_{(a) d}^{2}=0\right)$ and absolute anticommutativity $\left(s_{d} s_{a d}+s_{a d} s_{d}=0\right)$ properties of the (anti-)co-BRST symmetry transformations $s_{(a) d}$ are deeply connected with such properties (i.e., $\partial_{\theta}{ }^{2}=$ $\partial_{\bar{\theta}}^{2}=0, \partial_{\theta} \partial_{\bar{\theta}}+\partial_{\bar{\theta}} \partial_{\theta}=0$ ) associated with the translational generators $\partial_{\theta}$ and $\partial_{\bar{\theta}}$ on the $(1,2)$-dimensional supermanifold on which our present theory is generalized. Thus, it is the supervariable approach to BRST formalism that provides geometrical meaning to the abstract mathematical properties (e.g., nilpotency and absolute anticommutativity) associated with the (anti-)BRST symmetry transformations (and corresponding (anti-)BRST charges). Furthermore, this formalism also provides the interrelationships between nilpotency and anticommutativity properties (as we will see in Section 6 of our present endeavor).

\section{Invariance of Lagrangian: Supervariable Approach}

First of all, we note that the starting Lagrangian (1) can be written in the following three different and distinct forms:

$$
\begin{aligned}
L_{b}= & \dot{r} p_{r}+\dot{\vartheta} p_{\vartheta}-\frac{p_{\vartheta}^{2}}{2 r^{2}}-\lambda(r-a) \\
& +s_{b}\left[-i \bar{C}\left\{\left(\dot{\lambda}-p_{r}\right)+\frac{b}{2}\right\}\right] \\
\equiv & \dot{r} p_{r}+\dot{\vartheta}_{p_{\vartheta}}-\frac{p_{\vartheta}^{2}}{2 r^{2}}-\lambda(r-a) \\
& +s_{a b}\left[+i C\left\{\left(\dot{\lambda}-p_{r}\right)+\frac{b}{2}\right\}\right] \\
\equiv & \dot{r} p_{r}+\dot{\vartheta}_{p_{\vartheta}}-\frac{p_{\vartheta}^{2}}{2 r^{2}}-\lambda(r-a) \\
& +s_{b} s_{a b}\left[\frac{i}{2}\left(\lambda^{2}-p_{r}^{2}\right)+\frac{C \bar{C}}{2}\right],
\end{aligned}
$$

where it is basically the gauge-fixing and Faddeev-Popov ghost terms that have been expressed in three different ways because the original Lagrangian for the rigid rotor (without the gauge-fixing and Faddeev-Popov ghost terms) is (see, e.g., [3] for details)

$$
L_{0}=\dot{r} p_{r}+\dot{\vartheta} p_{\vartheta}-\frac{p_{\vartheta}^{2}}{2 r^{2}}-\lambda(r-a) .
$$

To be precise, all the above three forms are interconnected because the top two forms can be obtained from the bottom relation if we exploit the absolute anticommutativity property $\left(s_{b} s_{a b}+s_{a b} s_{b}=0\right)$ of the nilpotent $\left(s_{(a) b}^{2}=0\right)$ (anti-)BRST symmetry transformations $s_{(a) b}$. Towards our main goal of expressing Lagrangian (1) in terms of the supervariables, obtained after the application of suitable restrictions, first of all, we generalize the original Lagrangian $L_{0}$ onto $(1,2)$-dimensional supermanifold as

$$
L_{0} \longrightarrow \widetilde{L}_{0}=\dot{r} P_{r}^{(b)}(t, \theta, \bar{\theta})+\dot{\vartheta} p_{\vartheta}-\frac{p_{\vartheta}^{2}}{2 r^{2}}-\Lambda^{(h)}(t, \theta, \bar{\theta})(r-a),
$$


where $P_{r}^{(b)}(t, \theta, \bar{\theta})$ and $\Lambda^{(h)}(t, \theta, \bar{\theta})$ are from (17) and (11). It is straightforward to note that the (anti-)BRST invariance of the action integral corresponding to this part of the Lagrangian can be captured in the following expressions:

$$
\begin{aligned}
\left.\frac{\partial}{\partial \theta}\left[\widetilde{L}_{0}\right]\right|_{\bar{\theta}=0} & =-\frac{d}{d t}[\bar{C}(r-a)] \Longleftrightarrow \\
s_{a b} L_{0} & =-\frac{d}{d t}[\bar{C}(r-a)], \\
\left.\frac{\partial}{\partial \bar{\theta}}\left[\widetilde{L}_{0}\right]\right|_{\theta=0} & =-\frac{d}{d t}[C(r-a)] \Longleftrightarrow \\
s_{b} L_{0} & =-\frac{d}{d t}[C(r-a)], \\
\frac{\partial}{\partial \bar{\theta}} \frac{\partial}{\partial \theta}\left[\widetilde{L}_{0}\right] & =-i \frac{d}{d t}[b(r-a)] \Longleftrightarrow \\
s_{b} s_{a b} L_{0} & =-i \frac{d}{d t}[b(r-a)],
\end{aligned}
$$

where inputs from expansions (11) and (17) have been taken into account. Furthermore, we note that the following supervariable generalizations are trivial:

$$
\begin{gathered}
r(t) \longrightarrow R(t, \theta, \bar{\theta})=r(t), \\
\vartheta(t) \longrightarrow \Theta(t, \theta, \bar{\theta})=\vartheta(t), \\
p_{\vartheta}(t) \longrightarrow P_{\vartheta}(t, \theta, \bar{\theta})=p_{\vartheta}(t),
\end{gathered}
$$

because of the fact that these variables do not transform under the (anti-)BRST symmetry transformations (i.e., $\left.s_{(a) b}\left[r, \vartheta, p_{\vartheta}\right]=0\right)$. In other words, there are no Grassmannian expansions for these variables when they are generalized onto (1,2)-dimensional supermanifold.

In the above expressions (cf. (38), (39)), we have captured the (anti-)BRST invariance of the starting Lagrangian $L_{0}$ for the rigid rotor in the language of supervariable approach. The gauge-fixing and Faddeev-Popov ghost terms of the starting Lagrangian (1),

$$
L_{\mathrm{gf}}+L_{\mathrm{FP}}=b\left(\dot{\lambda}-p_{r}\right)+\frac{b^{2}}{2}-i \dot{\bar{C}} \dot{C}+i \bar{C} C,
$$

can be generalized onto the $(1,2)$-dimensional supermanifold as

$$
\begin{aligned}
\widetilde{L}_{\mathrm{gf}}+\widetilde{L}_{\mathrm{FP}}= & b(t)\left[\dot{\Lambda}^{(h)}-P_{r}^{(b)}\right]+\frac{b^{2}(t)}{2}-i \dot{\bar{F}}^{(h)} \dot{F}^{(h)} \\
& +i \bar{F}^{(h)} F^{(h)},
\end{aligned}
$$

where we have taken $b(t) \rightarrow B(t, \theta, \bar{\theta})=b(t)$ and the other expansions are given in (11) and (17). It is straightforward to check that

$$
\begin{aligned}
& \left.\frac{\partial}{\partial \theta}\left[\widetilde{L}_{\mathrm{gf}}+\widetilde{L}_{\mathrm{FP}}\right]\right|_{\bar{\theta}=0}=\frac{d}{d t}[b \dot{\bar{C}}] \equiv s_{a b}\left[L_{\mathrm{gf}}+L_{\mathrm{FP}}\right], \\
& \left.\frac{\partial}{\partial \bar{\theta}}\left[\widetilde{L}_{\mathrm{gf}}+\widetilde{L}_{\mathrm{FP}}\right]\right|_{\theta=0}=\frac{d}{d t}[b \dot{C}] \equiv s_{b}\left[L_{\mathrm{gf}}+L_{\mathrm{FP}}\right], \\
& \frac{\partial}{\partial \theta} \frac{\partial}{\partial \bar{\theta}}\left[\widetilde{L}_{\mathrm{gf}}+\widetilde{L}_{\mathrm{FP}}\right]=\frac{d}{d t}[i b \dot{b}] \equiv s_{b} s_{a b}\left[L_{\mathrm{gf}}+L_{\mathrm{FP}}\right] .
\end{aligned}
$$

Hence, the total (anti-)BRST invariant Lagrangian $L_{b}=L_{0}+$ $L_{\mathrm{gf}}+L_{\mathrm{FP}}$ can be expressed as the sum of (38) and (42) in the supervariable approach (as $\widetilde{L}_{b}=\widetilde{L}_{0}+\widetilde{L}_{\mathrm{gf}}+\widetilde{L}_{\mathrm{FP}}$ ). Now, it is straightforward to check that the following are true:

$$
\begin{aligned}
& \left.\frac{\partial}{\partial \overline{\bar{\theta}}}\left[\widetilde{L}_{b}\right]\right|_{\theta=0}=\frac{d}{d t}[b \dot{C}-C(r-a)] \equiv s_{b}\left[L_{0}\right], \\
& \left.\frac{\partial}{\partial \theta}\left[\widetilde{L}_{b}\right]\right|_{\bar{\theta}=0}=\frac{d}{d t}[b \dot{\bar{C}}-\bar{C}(r-a)] \equiv s_{a b}\left[L_{0}\right], \\
& \frac{\partial}{\partial \bar{\theta}} \frac{\partial}{\partial \theta}\left[\widetilde{L}_{b}\right]=\frac{d}{d t}[i b\{\dot{b}-(r-a)\}] \equiv s_{b} s_{a b}\left[L_{0}\right] .
\end{aligned}
$$

Thus, we have captured the (anti-)BRST invariance of the action $S=\int d t L_{b}$ in the language of supervariables (11) and (17) (obtained after various appropriate restrictions) and Grassmannian derivatives.

Taking the help of mappings in (19) and expansions in (11) and (17), it is straightforward to express Lagrangian (36) in the language of supervariable on the $(1,2)$-dimensional supermanifold; namely,

$$
\begin{aligned}
& L_{b} \longrightarrow \widetilde{L}_{b} \equiv \widetilde{L}_{0}+\left.\frac{\partial}{\partial \bar{\theta}}\left[-i \bar{F}^{(h)}\left\{\left(\dot{\Lambda}^{(h)}-P_{r}^{(b)}\right)+\frac{b(t)}{2}\right\}\right]\right|_{\theta=0}, \\
& L_{b} \longrightarrow \widetilde{L}_{b} \equiv \widetilde{L}_{0}+\left.\frac{\partial}{\partial \theta}\left[i F^{(h)}\left\{\left(\dot{\Lambda}^{(h)}-P_{r}^{(b)}\right)+\frac{b(t)}{2}\right\}\right]\right|_{\bar{\theta}=0}, \\
& L_{b} \longrightarrow \widetilde{L}_{b} \equiv \widetilde{L}_{0}+\frac{\partial}{\partial \bar{\theta}} \frac{\partial}{\partial \theta}\left[\frac{i}{2}\left(\Lambda^{(h)} \Lambda^{(h)}-P_{r}^{(b)} P_{r}^{(b)}\right)+\frac{F^{(h)} \bar{F}^{(h)}}{2}\right] .
\end{aligned}
$$


Using the nilpotency and anticommutativity properties of the translational generators $\left(\partial_{\theta}, \partial_{\bar{\theta}}\right)$, it is clear that the (anti-)BRST invariance of the action integral corresponding to the Lagrangian $L_{b}$ can be captured in the language of supervariable approach because $\left(\partial_{\theta} \widetilde{L}_{b}\right),\left(\partial_{\bar{\theta}} \widetilde{L}_{b}\right)$, and $\left(\partial_{\bar{\theta}} \partial_{\theta} \widetilde{L}_{b}\right)$ are all total time derivatives.
We concentrate now on the (anti-)co-BRST invariance of Lagrangian (1) in the language of the supervariable approach. Here, we will not be as much elaborate as we have been in the case of (anti-)BRST invariance of the Lagrangian within the framework of supervariable approach. We can generalize Lagrangian (1) to the $(1,2)$-dimensional supermanifold in a straightforward manner as

$$
L_{b} \longrightarrow \widetilde{L}_{b}^{(d)}=\dot{r} P_{r}^{(d)}+\dot{\vartheta} p_{\vartheta}-\frac{p_{\vartheta}^{2}}{2 r^{2}}-\Lambda^{(d)}(r-a)+b\left(\dot{\Lambda}^{(d)}-P_{r}^{(d)}\right)+\frac{b^{2}}{2}-i \dot{\bar{F}}^{(d)} \dot{F}^{(d)}+i \bar{F}^{(d)} F^{(d)}
$$

where $\left(\Lambda^{(d)}, P_{r}^{(d)}, F^{(d)}, \bar{F}^{(d)}\right)$ are expansions (34) that have been derived by exploiting the DHC (cf. (21)) and (anti-)coBRST invariant restrictions. The (anti-)co-BRST invariance of the starting Lagrangian (1) can be captured within the framework of supervariable approach, in the following fashion:

$$
\begin{aligned}
& \left.\frac{\partial}{\partial \bar{\theta}}\left[\widetilde{L}_{b}^{(d)}\right]\right|_{\theta=0}=0 \Longleftrightarrow s_{d}\left[L_{b}\right]=0, \\
& \left.\frac{\partial}{\partial \theta}\left[\widetilde{L}_{b}^{(d)}\right]\right|_{\bar{\theta}=0}=0 \Longleftrightarrow s_{a d}\left[L_{b}\right]=0, \\
& \frac{\partial}{\partial \bar{\theta}} \frac{\partial}{\partial \theta}\left[\widetilde{L}_{b}^{(d)}\right]=0 \Longleftrightarrow s_{d} s_{a d}\left[L_{b}\right]=0 .
\end{aligned}
$$

Geometrically, the (anti-)co-BRST invariance can be explained as follows. The super-Lagrangian $\widetilde{L}_{b}^{(d)}$ is the sum of composite (super)variables (obtained after DHC and appropriate set of (anti-)co-BRST invariant restrictions) such that its translation along $\theta$ and $\bar{\theta}$-directions yields zero result (which is equivalent to $s_{(a) d} L_{0}=0$ ).

\section{Nilpotency and Anticommutativity: Supervariable Approach to a 1D Rigid Rotor}

In this section, we discuss in detail the nilpotency and absolute anticommutativity of the (anti-)co-BRST charges within the framework of supervariable approach. We also briefly mention the same properties that are associated with the (anti-)BRST charges because this has been already discussed, to some extent, in our earlier work [4]. In fact, we will pinpoint only a few subtle points connected with the (anti-)BRST charges which have not been mentioned in our earlier work [4]. For instance, we will touch upon the absolute anticommutativity of the BRST and anti-BRST charges and its geometrical meaning in the language of the translational generators $\left(\partial_{\theta}, \partial_{\bar{\theta}}\right)$.

To begin with, first of all, we note that the (anti-)co-BRST charges $\left(Q_{(a) d}\right)$ can be expressed in the following forms within the framework of the supervariable approach:

$$
\begin{aligned}
Q_{a d} & =-i \frac{\partial}{\partial \bar{\theta}} \frac{\partial}{\partial \theta}\left[\dot{\Lambda}^{(d)} F^{(d)}\right] \\
& \equiv-i \int d \bar{\theta} \int d \theta\left[\dot{\Lambda}^{(d)} F^{(d)}\right],
\end{aligned}
$$

$$
\begin{aligned}
Q_{a d} & =-\left.i \frac{\partial}{\partial \bar{\theta}}\left[\dot{F}^{(d)} F^{(d)}\right]\right|_{\theta=0} \equiv-\left.i \int d \bar{\theta}\left[\dot{F}^{(d)} F^{(d)}\right]\right|_{\theta=0}, \\
Q_{a d} & =\left.i \frac{\partial}{\partial \theta}\left[\dot{\bar{F}}^{(d)} F^{(d)}-i \dot{\Lambda}^{(d)} \dot{b}(t)\right]\right|_{\bar{\theta}=0} \\
& \left.\equiv i \int d \theta\left[\dot{\bar{F}}^{(d)} F^{(d)}-i \dot{\Lambda}^{(d)} \dot{b}(t)\right]\right|_{\bar{\theta}=0}, \\
Q_{d} & =-i \frac{\partial}{\partial \bar{\theta}} \frac{\partial}{\partial \theta}\left[\dot{\Lambda}^{(d)} \bar{F}^{(d)}\right] \\
& \equiv-i \int d \bar{\theta}\left[d \theta\left[\dot{\Lambda}^{(d)} \bar{F}^{(d)}\right],\right. \\
Q_{d} & =\left.\left.i \frac{\partial}{\partial \bar{\theta}}\left[\dot{\bar{F}}^{(d)} \bar{F}^{(d)}\right]\right|_{\theta=0} \equiv i \int d \bar{\theta}\left[\dot{\bar{F}}^{(d)} \bar{F}^{(d)}\right]\right|_{\theta=0}, \\
Q_{d} & =-\left.i \frac{\partial}{\partial \theta}\left[\dot{F}^{(d)} \bar{F}^{(d)}+i \dot{\Lambda}^{(d)} \dot{b}(t)\right]\right|_{\bar{\theta}=0} \\
& \equiv-\left.i \int d \theta\left[\dot{F}^{(d)} \bar{F}^{(d)}+i \dot{\Lambda}^{(d)} \dot{b}(t)\right]\right|_{\bar{\theta}=0},
\end{aligned}
$$

where superexpansions (34) have been taken into account which have been derived after the application of the DHC (cf. (21)) and several other (anti-)co-BRST invariant restrictions. Furthermore, consistent with superexpansions (34), the (anti-)co-BRST charges in (5) have been reexpressed as follows:

$$
\begin{aligned}
Q_{d} & =\dot{r} \bar{C}-(r-a) \dot{\bar{C}}, \\
Q_{a d} & =\dot{r} C-(r-a) \dot{C},
\end{aligned}
$$

where we have used the Euler-Lagrange equations of motion $b=\dot{r}$ and $\dot{b}=-(r-a)$ that emerge from the starting Lagrangian (1) because of the least action principle. There are some alternative expressions for the ones quoted in (48). For instance, one can replace $\dot{\Lambda}^{(d)}$ by $P_{r}^{(d)}$ and, once again, we obtain the same expressions for $Q_{(a) d}$.

Due to the mappings listed in (35), we can express expressions (48) in the ordinary space in the language of 
the nilpotent and absolutely anticommuting (anti-)co-BRST transformations $s_{a d}$ and ordinary variables as

$$
\begin{aligned}
Q_{a d} & =-i s_{d} s_{a d}[\dot{\lambda} C], \\
Q_{d} & =-i s_{d} s_{a d}[\dot{\lambda} \bar{C}], \\
Q_{a d} & =-i s_{d}[\dot{C} C], \\
Q_{d} & =i s_{a d}[\dot{\bar{C}} \bar{C}], \\
Q_{a d} & =i s_{a d}[\dot{\bar{C}} C-i \dot{\lambda} \dot{b}], \\
Q_{d} & =-i s_{d}[\dot{C} \bar{C}+i \dot{\lambda} \dot{b}] .
\end{aligned}
$$

By exploiting the (anti-)co-BRST symmetry transformation (4), it can be checked that the above expressions do match with (49) (which is also equivalent to expressions given in (5) in terms of the auxiliary variable $b(t))$. From the above equations, it becomes transparent that the nilpotency of (anti-) co-BRST charges is deeply connected with the nilpotency $\left(s_{(a) d}^{2}=0\right)$ of (anti-)co-BRST symmetry transformations as well as the nilpotency $\left(\partial_{\theta}^{2}=0, \partial_{\bar{\theta}}^{2}=0\right)$ of the translational generators $\partial_{\theta}$ and $\partial_{\bar{\theta}}$ along the Grassmannian directions of this $(1,2)$-dimensional supermanifold. For instance, if we consider $Q_{d}=i s_{a d}[\dot{\bar{C}} \bar{C}]$, it is clear that $s_{d} Q_{d}=i\left\{Q_{d}, Q_{d}\right\}=0$ because of $s_{d}^{2}=0$ and the basic definition of a generator of a given transformation. Furthermore, from the suitable expressions from (48), it is very evident that $\partial_{\bar{\theta}} Q_{d}=0$ due to $\partial_{\bar{\theta}}^{2}=0$ which, in turn, implies that $Q_{d}^{2}=0$. Such kind of arguments can be also given for the nilpotency of $Q_{a d}$ as well. Geometrically, the equation $Q_{d}=\left.\partial_{\bar{\theta}}\left[\dot{\bar{F}}^{(d)} \bar{F}^{(d)}\right]\right|_{\theta=0}$ implies that the co-BRST charge $Q_{d}$ is already equivalent to the translation of a composite supervariable $\left(\dot{\bar{F}}^{(d)} \bar{F}^{(d)}\right)$ along the $\bar{\theta}$-direction of the supermanifold. Thus, any further translation along $\bar{\theta}$-direction produces a zero result because of the fermionic $\left(\partial_{\bar{\theta}}^{2}=0\right)$ nature of $\partial_{\bar{\theta}}$. Similar explanation for the nilpotency of the suitable expression for $Q_{a d}$ can be given in the language of nilpotency $\left(\partial_{\theta}^{2}=0\right)$ of the translational generator $\partial_{\theta}$ along $\theta$-direction.

Now we dwell a bit on the geometrical meaning of the absolute anticommutativity of the (anti-)co-BRST charges $Q_{a d}$ in the language of the translational generators $\left(\partial_{\theta}\right.$ and $\left.\partial_{\bar{\theta}}\right)$ along the Grassmannian directions of the supermanifold. Let us take the example

$$
\begin{aligned}
Q_{d} & =-\left.i \frac{\partial}{\partial \theta}\left[\dot{F}^{(d)} \bar{F}^{(d)}+i \dot{\Lambda}^{(d)} \dot{b}(t)\right]\right|_{\bar{\theta}=0} \\
& \equiv-i s_{a d}[\dot{C} \bar{C}+i \dot{\lambda} \dot{b}] .
\end{aligned}
$$

It is self-evident that $s_{a d} Q_{d}=0$ because of the nilpotency $\left(s_{a d}^{2}=0\right)$ of $s_{a d}$ and $\partial_{\theta} Q_{d}=0$ because of the nilpotency $\left(\partial_{\theta}^{2}=\right.$ $0)$ of the translational generator $\partial_{\theta}$. However, if we take the definition of the generator for the transformation $s_{a d}$, then, $s_{a d} Q_{d}=i\left\{Q_{d}, Q_{a d}\right\}=0$ due to the nilpotency $\left(s_{a d}^{2}=0\right)$ of $s_{a d}$ which in turn implies the absolute anticommutativity of the (anti-)co-BRST charges $Q_{(a) d}$. The other expression for $Q_{d}$ (e.g., $Q_{d}=\left.\partial_{\bar{\theta}}\left[i \dot{\bar{F}}^{(d)} F^{(d)}\right]\right|_{\theta=0}$ ) implies that $\partial_{\bar{\theta}} Q_{d}=0$. If we operate by $\partial_{\bar{\theta}}$ on (51), we should get $\partial_{\bar{\theta}} Q_{d}=0$. However, it leads to the following explicit expressions:

$$
\begin{aligned}
\frac{\partial}{\partial \bar{\theta}} Q_{d} & =0=-i \frac{\partial}{\partial \bar{\theta}} \frac{\partial}{\partial \theta}\left[\dot{F}^{(d)} \bar{F}^{(d)}+i \dot{\Lambda}^{(d)} \dot{b}(t)\right] \\
& \equiv-\frac{i}{2}\left(\partial_{\theta} \partial_{\bar{\theta}}+\partial_{\bar{\theta}} \partial_{\theta}\right)\left[\dot{F}^{(d)} \bar{F}^{(d)}+i \dot{\Lambda}^{(d)} \dot{b}(t)\right],
\end{aligned}
$$

which shows the absolute anticommutativity of the (anti-)coBRST charges because of the fact that $\partial_{\theta} \partial_{\bar{\theta}}+\partial_{\bar{\theta}} \partial_{\theta}=0$. If we take into account the mappings listed in (35), we obtain $s_{d} s_{a d}+s_{a d} s_{d}=0$. The latter is equivalent to the absolute anticommutativity of (anti-)co-BRST charges. On the other hand, from (51), it is clear that $\partial_{\theta} Q_{d}=0$ because of the nilpotency of $\partial_{\theta}$ (i.e., $\partial_{\theta}^{2}=0$ ). Thus, we observe that the nilpotency and anticommutativity are interrelated. These observations are true because the nilpotency condition $\left(\partial_{\theta}^{2}=\partial_{\bar{\theta}}^{2}=0\right)$ is a limiting case of the absolute anticommutativity $\left(\partial_{\theta} \partial_{\bar{\theta}}+\partial_{\bar{\theta}} \partial_{\theta}=\right.$ 0 ) because of the fact that (i) when we take $\partial_{\theta}=\partial_{\bar{\theta}}$, we obtain $\partial_{\bar{\theta}}^{2}=0$, and (ii) when we choose $\partial_{\bar{\theta}}=\partial_{\theta}$, we get $\partial_{\theta}^{2}=0$. Similar inferences could be drawn for the nilpotency $\left(s_{a(d)}^{2}=\right.$ $0)$ of the (anti-)co-BRST symmetry transformations $\left(s_{(a) d}\right)$ (and their corresponding charges $Q_{(a) d}$ ) from the absolute anticommutativity $s_{d} s_{a d}+s_{a d} s_{d}=0$ (and their counterparts $\left.Q_{d} Q_{a d}+Q_{a d} Q_{d}=0\right)$.

We close this section with a brief remark about the absolute anticommutativity $\left(s_{b} s_{a b}+s_{a b} s_{b}=0, Q_{b} Q_{a b}+Q_{a b} Q_{b}=\right.$ 0 ) of the (anti-)BRST symmetries (and their corresponding charges $\left.Q_{(a) b}\right)$ which have been discussed in our earlier work [4] within the framework of supervariable approach. For instance, we have obtained the results $Q_{b}=i s_{a b}(C \dot{C})$, $Q_{a b}=-i s_{b}(\bar{C} \dot{\bar{C}})$, and their corresponding expressions in supervariable approach. Now, it is crystal clear that $s_{a b} Q_{b}=$ $i\left\{Q_{b}, Q_{a b}\right\}=0$ due to the nilpotency $\left(s_{a b}^{2}=0\right)$ of $s_{a b}$. Similarly, $s_{b} Q_{a b}=i\left\{Q_{a b}, Q_{b}\right\}=0$ due to the nilpotency $\left(s_{b}^{2}=0\right)$ of the BRST symmetry transformations $s_{b}$. Thus, we note that the absolute anticommutativity of (anti-)BRST charges is connected with the nilpotency $\left(s_{(a) b}^{2}=0\right)$ of the (anti-)BRST symmetry transformations $s_{(a) b}$. These observations are $\log$ ical because as discussed earlier, the absolute anticommutativity $\left(\partial_{\theta} \partial_{\bar{\theta}}+\partial_{\bar{\theta}} \partial_{\theta}=0\right)$ of the translational generators $\left(\partial_{\theta}, \partial_{\bar{\theta}}\right)$ is connected with the nilpotency $\left(\partial_{\theta}^{2}=0=\partial_{\bar{\theta}}^{2}\right)$ of these translational operators which is the limiting cases when $\partial_{\theta}=$ $\partial_{\bar{\theta}}$ and $/$ or $\partial_{\bar{\theta}}=\partial_{\theta}$.

\section{Conclusions}

In our present endeavor, we have derived the (anti-)BRST symmetry transformations by exploiting the ideas of (i) horizontality condition and (ii) (anti-)BRST invariant restrictions, on the supervariables which are defined on the suitably chosen (1, 2)-dimensional supermanifold (on which our ordinary theory is generalized). These ideas are geometrically and physically more intuitive as well as elegant and the latter 
condition is completely different from our earlier work [4] where mathematically correct (but ad-hoc) approximations have been made. In our present investigation, the geometrical interpretation for the nilpotency and anticommutativity properties, associated with the (anti-)BRST charges, remain the same as what has been discussed in our earlier work [4] on this topic.

One of the relatively novel features of our present investigation is the systematic application of the DHC for the precise derivation of the proper (anti-)co-BRST symmetry transformations where the Hodge duality $(\star)$ operation on the $(1,2)$-dimensional supermanifold plays a very decisive role. We have verified that the working-rules, laid down in [14], turn out to be correct because we are able to derive the precise form of the nilpotent (anti-)co-BRST symmetry transformations in a consistent manner. We have also provided the geometrical basis for the (anti-)co-BRST charges in the language of the supervariables (obtained after the application of the appropriate set of restrictions) and the translational generators along the Grassmannian directions of the supermanifold.

It is very important for us to apply the key ideas of DHC (and associated Hodge duality $\star$ operation) in the context of the other higher dimensional physical systems of interest (that have been proven to be the tractable physical examples of Hodge theory) so that the working-rules, laid down in [14], could be tested on any arbitrary (D, 2)dimensional supermanifold. For instance, we have already discussed the utility of the Hodge duality $\star$ operation on the $(4,2)$-dimensional supermanifold in the case of 4D Abelian gauge theory in our earlier work [14]. Thus, the application of the DHC (in the context of some physical systems of interest) remains a central issue for our future endeavors. It is gratifying to state that we have already applied the DHC in the cases of the modified versions of 2D Proca theory as well as the chiral bosonic field theory and have obtained the precise form of the (anti-)co-BRST symmetries $[15,16]$. We are currently busy with the ideas of the application of DHC and our results would be reported in our future publications.

\section{Appendix}

We compute here the explicit expression for $\star \widetilde{d} \star \tilde{\lambda}^{(1)}$ which has been used in the DHC (21). Towards this goal in mind, we exploit the working-rule, developed in [14], for the Hodge duality operation on a $(1,2)$-dimensional supermanifold. To begin with, we have the following single $(*)$ operation on the super 1-form:

$$
\star \tilde{\lambda}^{(1)}=\star(d t \Lambda+d \theta F+d \bar{\theta} F) .
$$

According to the working-rule laid down in [14], we have the following correct $(*)$ operation on the 1-form differentials of the $(1,2)$-dimensional supermanifold:

$$
\begin{aligned}
\star(d t) & =(d \theta \wedge d \bar{\theta}), \\
\star(d \theta) & =(d t \wedge d \bar{\theta}), \\
\star(d \bar{\theta}) & =(d t \wedge d \theta) .
\end{aligned}
$$

The above expressions physically imply that, on the $(1,2)$ dimensional supermanifold, the dual of the differential $(d t)$ is $(d \theta \wedge d \bar{\theta})$. In exactly a similar fashion, the dual of the differentials $(d \theta)$ and $(d \bar{\theta})$ has been expressed (taking into account the physical arguments). These inputs imply the following expression for the super 2-form that is derived from (A.1):

$$
\star \tilde{\lambda}^{(1)}=(d \theta \wedge d \bar{\theta}) \Lambda+(d t \wedge d \bar{\theta}) F+(d t \wedge d \theta) F
$$

Now, we have to operate $\widetilde{d}=d t \partial_{t}+d \theta \partial_{\theta}+d \bar{\theta} \partial_{\bar{\theta}}$ on it. As a consequence of this operation, we obtain the following super 3-form:

$$
\begin{aligned}
\tilde{d} \star \tilde{\lambda}^{(1)}= & (d t \wedge d \theta \wedge d \bar{\theta}) \dot{\Lambda}+(d t \wedge d \bar{\theta} \wedge d t) \dot{\bar{F}} \\
& +(d t \wedge d t \wedge d \theta) \dot{F}+(d \theta \wedge d \theta \wedge d \bar{\theta}) \partial_{\theta} \lambda \\
& -(d \theta \wedge d t \wedge d \bar{\theta}) \partial_{\theta} \bar{F} \\
& -(d \theta \wedge d t \wedge d \theta) \partial_{\theta} F \\
& +(d \bar{\theta} \wedge d \theta \wedge d \bar{\theta}) \partial_{\bar{\theta}} \lambda \\
& -(d \bar{\theta} \wedge d t \wedge d \bar{\theta}) \partial_{\bar{\theta}} \bar{F} \\
& -(d \bar{\theta} \wedge d t \wedge d \theta) \partial_{\bar{\theta}} F
\end{aligned}
$$

To fully calculate $\star \widetilde{d} \star \tilde{\lambda}^{(1)}$, we have to operate another $(\star)$ on the above super 3 -form to obtain a super 0 -form. Before we carry out the above operation, it is clear that the second and third terms of the top line in (A.4) would be zero due to $(d t \wedge$ $d t=0)$. Further, as the working-rules laid down in [14], the 3-forms with only Grassmannian differentials would be zero on the $(1,2)$-dimensional supermanifold because physically such a supermanifold cannot accommodate a super 3-form that is expressed in terms of the wedge products of three Grassmannian variables only. Physically, the allowed super 3form differential wedge products on the $(1,2)$-dimensional supermanifold are $(d t \wedge d \theta \wedge d \bar{\theta}),(d t \wedge d \theta \wedge d \theta),(d t \wedge d \bar{\theta} \wedge d \bar{\theta})$ because these contain the wedge products that incorporate one differential $(d t)$ of bosonic nature and two differentials [i.e., $(d \theta \wedge d \theta),(d \theta \wedge d \bar{\theta})$ and $(d \bar{\theta} \wedge d \bar{\theta})]$ of the fermionic nature. These arguments imply that the fourth and seventh terms would be zero. To be more precise, we note that the coefficients of 3 -form differential wedge products $(d \theta \wedge d \theta \wedge$ $d \bar{\theta})$ and $(d \bar{\theta} \wedge d \theta \wedge d \bar{\theta})$ do not contribute to the derivation of the proper (anti-)co-BRST symmetries. Thus, these terms are not physically important. As a consequence, only the following terms would, ultimately, exist in (A.4):

$$
\begin{aligned}
\tilde{d} \star \tilde{\lambda}^{(1)}= & (d t \wedge d \theta \wedge d \bar{\theta}) \dot{\Lambda}+(d t \wedge d \theta \wedge d \bar{\theta}) \partial_{\theta} \bar{F} \\
& +(d t \wedge d \theta \wedge d \theta) \partial_{\theta} F \\
& +(d t \wedge d \bar{\theta} \wedge d \bar{\theta}) \partial_{\bar{\theta}} \bar{F} \\
& +(d t \wedge d \theta \wedge d \bar{\theta}) \partial_{\bar{\theta}} F
\end{aligned}
$$


It is worth pointing out that, mathematically, any arbitrary number of differentials may exist in the wedge product with only the Grassmannian differentials (e.g., $d \theta \wedge d \theta \wedge$ $d \theta \wedge d \theta, d \bar{\theta} \wedge d \bar{\theta} \wedge d \bar{\theta} \wedge d \bar{\theta} \cdots)$ and so forth. However, physically, it is not permitted to have any arbitrary number of wedge products of the Grassmannian differentials on a given finite $(D, 2)$-dimensional supermanifold on which a $D$ dimensional ordinary physical theory is generalized. Thus, the derivation of (A.5) is physically correct. Now, the stage is set to apply another $(*)$ on it. Using the following inputs (see, e.g., [14]) on the $(1,2)$-dimensional supermanifold

$$
\begin{aligned}
& \star(d t \wedge d \theta \wedge d \bar{\theta})=1, \\
& \star(d t \wedge d \theta \wedge d \theta)=s^{\theta \theta}, \\
& \star(d t \wedge d \bar{\theta} \wedge d \bar{\theta})=s^{\bar{\theta} \bar{\theta}},
\end{aligned}
$$

where $s^{\theta \theta}$ and $s^{\bar{\theta} \bar{\theta}}$ are symmetric in $\theta$ and $\bar{\theta}$ indices, we obtain the final expression

$$
\star \widetilde{d} \star \tilde{\lambda}^{(1)}=\left(\dot{\lambda}+\partial_{\theta} \bar{F}+\partial_{\bar{\theta}} F\right)+s^{\bar{\theta}} \bar{\theta} \partial_{\bar{\theta}} \bar{F}+s^{\theta \theta} \partial_{\theta} F
$$

which is used in the main body of our text (cf. (22)). The first entry of (A.6) physically implies that the dual of the wedge product $(d t \wedge d \theta \wedge d \bar{\theta})$ is nothing but unity (i.e., a 0 -form) as all the three independent differentials of the $(1,2)$-dimensional supermanifold are present in it. On the other hand, the dual of $(d t \wedge d \theta \wedge d \theta)$ has been taken to be $s^{\theta \theta}$ (i.e., a 0 -form) because when we take another $(*)$ operation on it, we should get back the original wedge product $(d t \wedge d \theta \wedge d \theta)$ modulo a sign factor. Similar is the argument for the definition of the duality operation on the super 3 -form wedge product $(d t \wedge d \bar{\theta} \wedge d \bar{\theta})$.

We would like to end this Appendix with the remarks that another Hodge duality ( $*$ ) operation on (A.2) is as follows:

$$
\begin{aligned}
& \star[\star(d t)]=\star(d \theta \wedge d \bar{\theta})=d t, \\
& \star[\star(d \theta)]=\star(d t \wedge d \bar{\theta})=d \theta, \\
& \star[\star(d \bar{\theta})]=\star(d t \wedge d \theta)=d \bar{\theta} .
\end{aligned}
$$

Physically, a single Hodge duality operation on the super 2form differentials $(d \theta \wedge d \bar{\theta})$ would be dual of this wedge product on a $(1,2)$-dimensional supermanifold. It is selfevident that it should be a 1 -form. Since the dual direction of $(\theta, \bar{\theta})$ is $t$ on a $(1,2)$-dimensional supermanifold, it is clear that the resulting 1 -form of the dual of $(d \theta \wedge d \bar{\theta})$ would be nothing but $d t$. Similar explanation can be given for the other double $(*)$ operations on the above 1-form differentials. We would like to lay emphasis on the importance of the factors

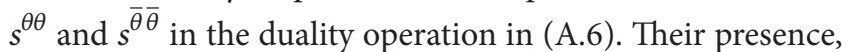
on the r.h.s., gives the idea that when we will take another $(\star)$ operation on the super 3 -forms (in (A.6)), we will get back the original super 3-forms (modulo some sign factors); namely,

$$
\begin{aligned}
& \star[\star(d t \wedge d \theta \wedge d \theta)]=\star s^{\theta \theta}=(d t \wedge d \theta \wedge d \theta), \\
& \star[\star(d t \wedge d \bar{\theta} \wedge d \bar{\theta})]=\star s^{\bar{\theta}}=(d t \wedge d \bar{\theta} \wedge d \bar{\theta}), \\
& \star[\star(d t \wedge d \theta \wedge d \bar{\theta})]=\star[1]=(d t \wedge d \theta \wedge d \bar{\theta}) .
\end{aligned}
$$

It is clear that the presence of $s^{\theta \theta}$ and $s^{\bar{\theta}} \bar{\theta} d o$ help us in getting the original super 3-forms after the application of a couple of successive Hodge duality operations. We have not got any sign factors on the r.h.s. (other than $(+)$ sign) because of the fact that we have discussed the double duality operations on a $(1,2)$-dimensional supermanifold. However, we do get $( \pm)$ signs, after the above kind of double duality operations, on the $(2,2)$-dimensional supermanifold (see, e.g., [14] for details). In a very recent work [17], the Hodge duality operation on a supermanifold has been discussed in a very elegant manner because of the fact that a whole lot of deep mathematical concepts have been taken into account. We are sure that the contents of this work [17] are important and they will be very useful for us in our future work (when we will take into account the supermanifolds which would not be necessarily flat). For our present endeavor, however, we feel that the material contained, in our earlier work [14] for the flat $(1,2)$ dimensional supermanifold, is good enough.

\section{Competing Interests}

The authors declare that there is no conflict of interests as far as the publication of this paper is concerned.

\section{Acknowledgments}

T. Bhanja is grateful to the BHU fellowship and D. Shukla thanks UGC, Government of India, New Delhi, for the financial support through RFSMS scheme under which the present investigation has been carried out.

\section{References}

[1] P. A. M. Dirac, Lectures on Quantum Mechanics, Belfer Graduate School of Science, Yeshiva University Press, New York, NY, USA, 1964.

[2] K. Sundermeyer, Constrained Dynamics, vol. 169 of Lecture Notes in Physics, Springer, Berlin, Germany, 1982.

[3] D. Nemeschansky, C. Preitschopf, and M. Weinstein, "A BRST primer," Annals of Physics, vol. 183, no. 2, pp. 226-268, 1988.

[4] S. Gupta and R. P. Malik, "Rigid rotor as a toy model for Hodge theory," European Physical Journal C, vol. 68, no. 1, pp. 325-335, 2010.

[5] T. Eguchi, P. B. Gilkey, and A. J. Hanson, "Gravitation, gauge theories and differential geometry," Physics Reports, vol. 66, no. 6, pp. 213-393, 1980.

[6] S. Mukhi and N. Mukunda, Introduction to Topology, Differential Geometry and Group Theory for Physicists, Wiley Eastern Pvt. Ltd, New Delhi, India, 1990. 
[7] J. W. van Holten, "Becchi-Rouet-Stora-Tyutin cohomology of compact gauge algebras," Physical Review Letters, vol. 64, article 2863, 1990.

[8] K. Nishijima, "Observable states in the representations of BRS algebra," Progress of Theoretical Physics, vol. 80, no. 5, pp. 905912, 1988.

[9] L. Bonora and M. Tonin, "Superfield formulation of extended BRS symmetry," Physics Letters B, vol. 98, no. 1-2, pp. 48-50, 1981.

[10] L. Bonora, P. Pasti, and M. Tonin, "Geometric description of extended BRS symmetry in superfield formulation," Il Nuovo Cimento A, vol. 63, no. 3, pp. 353-364, 1981.

[11] R. Delbourgo and P. D. Jarvis, "Extended BRS invariance and OSp (4/2) supersymmetry," Journal of Physics A: Mathematical and General, vol. 15, no. 2, pp. 611-625, 1982.

[12] R. Delbourgo, P. D. Jarvis, and G. Thompson, "Local OSp(4/2) supersymmetry and extended BRS transformations for gravity," Physics Letters B, vol. 109, no. 1-2, pp. 25-27, 1982.

[13] N. Nakanishi and I. Ojima, Covariant Operator Formalism of Gauge Theories and Quantum Gravity, World Scientific, Singapore, 1990.

[14] R. P. Malik, "Hodge duality operation and its physical applications on supermanifolds," International Journal of Modern Physics A, vol. 21, no. 16, pp. 3307-3336, 2006.

[15] A. Shukla, S. Krishna, and R. P. Malik, "Augmented superfield approach to nilpotent symmetries of the modified version of $2 \mathrm{D}$ Proca theory," Advances in High Energy Physics, vol. 2015, Article ID 258536, 21 pages, 2015.

[16] D. Shukla, T. Bhanja, and R. P. Malik, "Self-dual chiral boson: augmented superfield approach," The European Physical Journal C, vol. 74, article 3025, 2014.

[17] L. Castellani, R. Catenacci, and P. A. Grassi, "The Hodge operator revisited," https://arxiv.org/abs/1511.05105. 

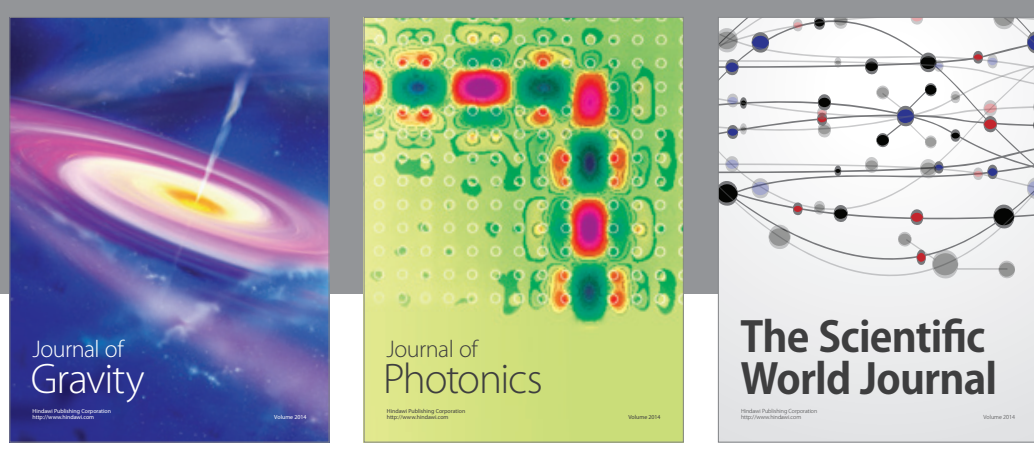

The Scientific World Journal
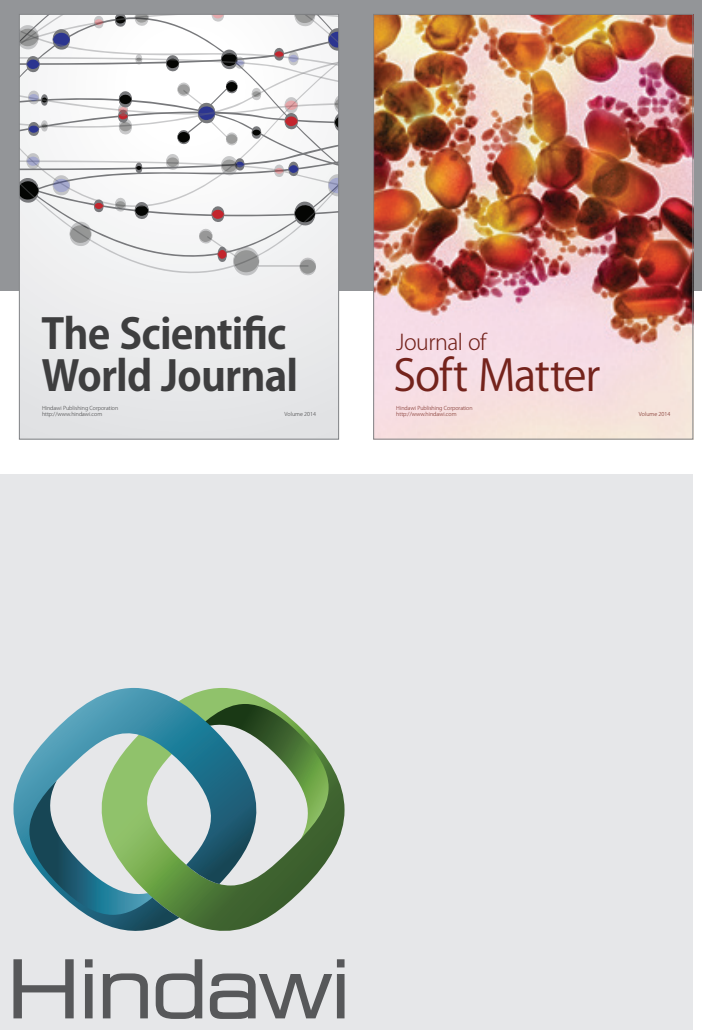

Submit your manuscripts at

http://www.hindawi.com

nternational Journal of

Statistical Mechanics
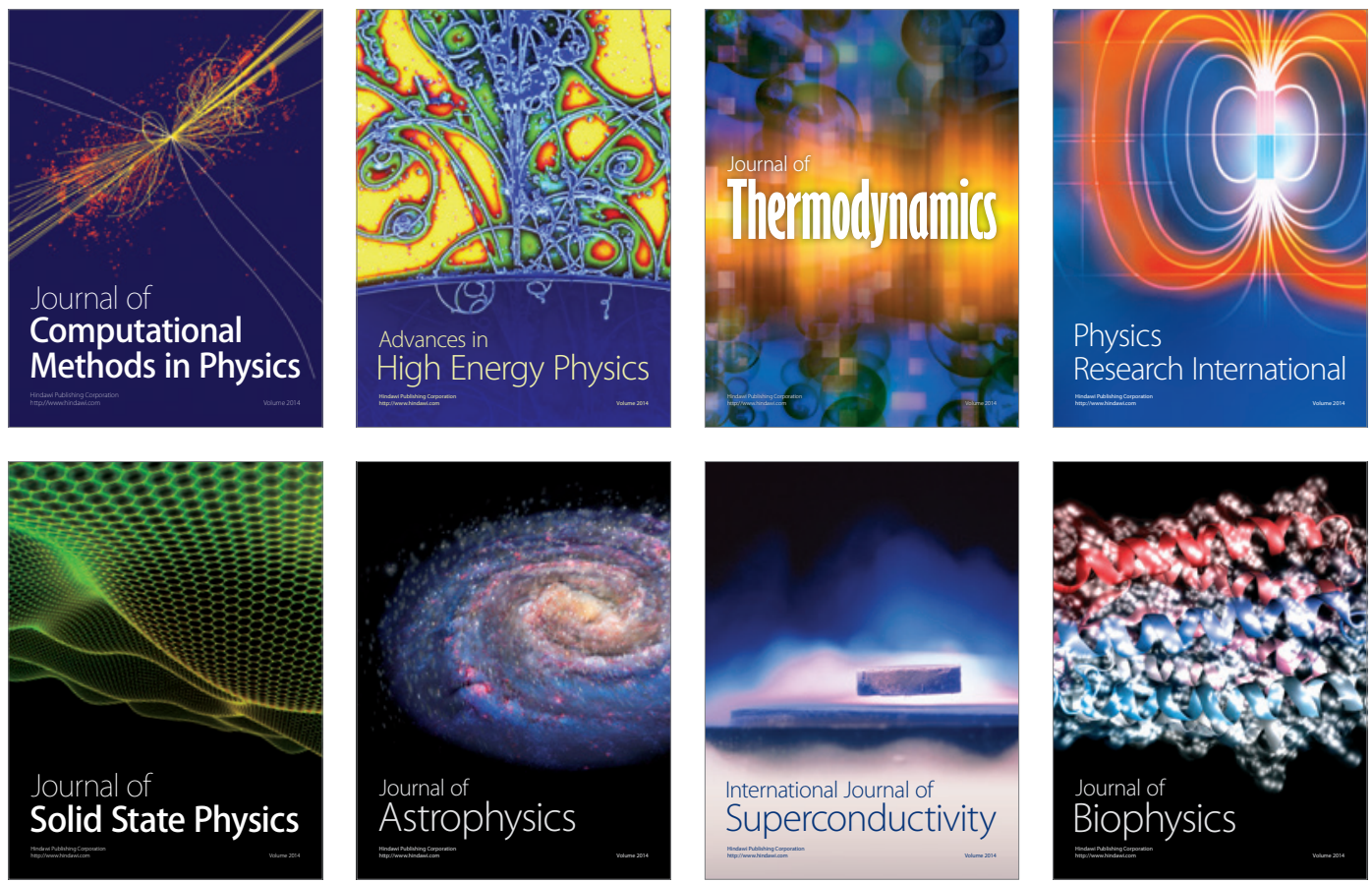
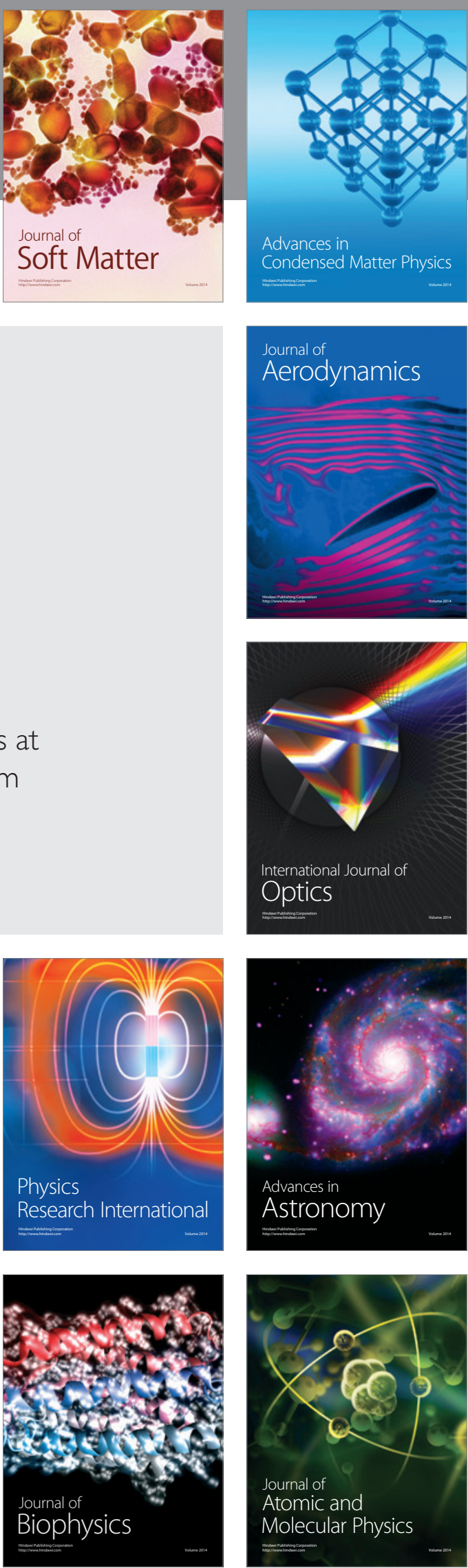